\title{
Real-time bit wear prediction using mud logger data with mathematical approaches
}

\author{
Hongpeng Yang ${ }^{1} \cdot$ Hongkai Zhao ${ }^{1} \cdot$ Suresh Kottapurath ${ }^{1}$
}

Received: 6 June 2019 / Accepted: 17 August 2019 / Published online: 23 August 2019

(c) The Author(s) 2019

\begin{abstract}
Nowadays, the success of drilling operations has become more sensitive to cost control. As one of the most effective cost reductions is the elimination of extra trips, real-time bit wear prediction has been a challenge for appropriate decision-making in drilling operations to reach the highest drilling performance and avoid serious bit issues. This present study shows the combination of mechanical specific energy (MSE), principal component analysis (PCA) and wavelet analysis in real-time bit wear prediction with mud logger data for the Kymera bit. The aforementioned mathematical approaches were combined with the traditional MSE method to monitor the drilling efficiency, especially to eliminate the loss of cone. Novel analytical approaches like PCA and wavelet analysis were introduced to predict the real-time bit wear grade for PDC part of the Kymera bit. Two field cases with several applications were selected for this study to illustrate the advantages of the aforementioned methods and demonstrate their efficiency in bit wear prediction. The first case study shows the bit wear prediction at pulling depth as Grade 5, while the actual dull grade of PDC part is Grade 5-5 without losing the cone. The second case study shows the bit wear prediction at the pulling depth is Grade 1, while the actual dull grade of PDC part is Grade 1-1 without losing the cone. The novelty of this bit wear prediction model is the ability to predict the Kymera bit wear in real time using mathematical approaches with practical applications and valid results in the mentioned study area.
\end{abstract}

Keywords Bit wear prediction · Principal component analysis · Wavelet analysis · Mechanical specific energy

\section{Introduction}

The objective of any drilling operation is the safe execution of drilling plans at an optimum performance by minimizing nonproductive time (NPT) with reducing the number of trips to sustain efficient drilling for as long as possible. The prediction of wear of the drill bit without the pull bit from the hole can improve efficiency in drilling operations. However, a decrease in the rate of penetration is not sufficient evidence as an indication of bit wear due to many scenarios such as bit balling and rock hardness increment. Mathematical models on the rate of penetration (ROP) with bit wear factor have been proposed (Bourgoyne and Young 1974). However, these models cannot be applied in realtime bit wear analysis (Rashidi and Hareland 2010). The most extensive usage of efficiency of the drilling bit is the

Hongpeng Yang

hong-peng.yang@shell.com

1 Shell China Exploration and Production Company, Beijing, China mechanical specific energy (MSE) technique. The concept of MSE for effect on bit performance was first introduced as the energy required for destroying a unit volume of rock by Teale (1965).

The method proposed in this study is based on principal component analysis (PCA) and wavelet analysis. The main feature in bit wear prediction is dimensionality reduction of the synthesized mud logger data using PCA, and subsequently, the wavelet analysis is applied to build the prediction model. In addition, the MSE method has been discussed as the auxiliary analysis to greatly increase the accuracy of bit wear prediction. Application of MSE method has been discussed, and a novel approach of numerical analysis has been introduced to perform real-time bit wear prediction.

\section{Methodology of MSE}

The concept of mechanical specific energy (MSE) is defined as the work required to destroy a given volume of the rock, which can be used as a competitive tool to detect 
changes in drilling efficiency and indicating real-time drilling issues. The basic approach of MSE is expressed in terms of drilling parameters to achieve the real-time calculation as follows:

$\mathrm{MSE}=\frac{\mathrm{WOB}}{A_{\mathrm{B}}}+\frac{120 \pi \times N \times T}{A_{\mathrm{B}} \times \mathrm{ROP}}$

In the above formula, $A_{\mathrm{B}}$ is bit surface area $\left(\right.$ inch $\left.^{2}\right), N$ is rotary speed (round per minute), and $T$ is the torque experienced by bit $(l b f \times f t)$ and MSE in $p s i$ (Teale 1965).

Bit torque has been estimated with a simple bit model considered as a cylindrical lump with the coefficient of friction $\mu$ as (Pessier et al. 1992):

$\mathrm{TOB}=\frac{2 r_{b}}{3} \mu \mathrm{WOB}$

Typically, friction $\mu$ is suggested between 0.6 and 2.0 for a PDC bit. However, it is hard to define the friction value in a study area, especially using the Kymera hybrid drill bit. Thus, bit torque has been normalized with surface torque using an experience model. Waughman introduced a methodology of separately MSE measurement in every section drilled combined with the current formation type to provide an indicator of the bit performance. This demonstrates a decision-making method on when to pull the bit by comparing instantaneous MSE values with the normal baseline (Waughman et al. 2003). Dupriest additionally stated that if the values of specific energy remain constant, then the bit is efficient; however, when MSE rises steadily higher than the usual values, a hazardous indication appears and must be mitigated (Dupriest and Koederitz 2005). Bit vibrations could certainly cause a similar increase in MSE values; however, surveillance of MSE with penetration rate can mitigate this confusion to a certain extent (Motahari et al. 2008). The MSE was calculated in Well A as shown in Fig. 1. The MSE baseline has been set up, and the result shows a sudden increase at $4220 \mathrm{~m}$ with bit dull grade of losing the cone and the bit totally worn out. This shows that MSE is a trustable indicator for severe bit wear. However, MSE method cannot precisely predict bit wear with a dull grade. Therefore, numerical analysis was introduced by reducing the dimension with PCA and data compression with wavelet analysis in the study area.

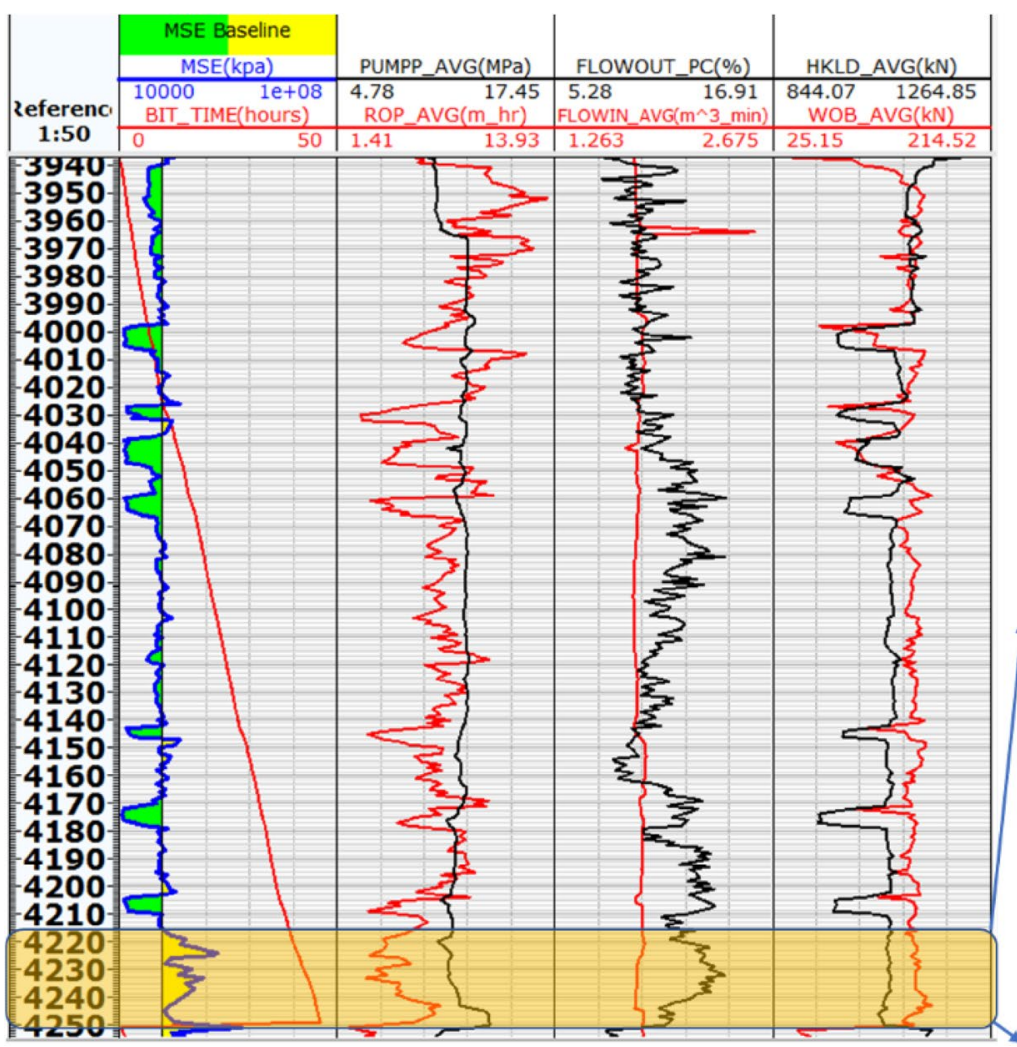

DULL GRADE:

8-8-CR-C/N-X-3-BT-PP(PDC)

8-8-LC-A-F-3-NO-PP (TCI)

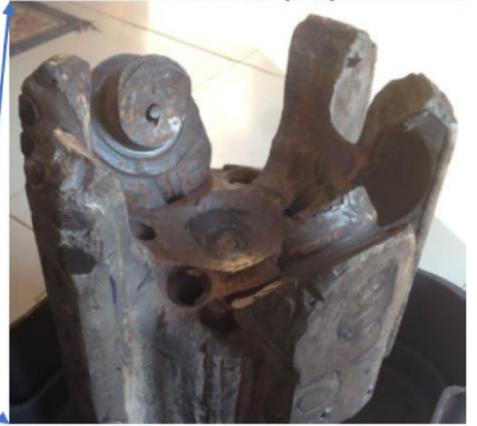

Fig. 1 Graphical representation of MSE and surface drilling parameter in Well A 


\section{Methodology of PCA and wavelet analysis}

Principal component analysis (PCA) is a method to create a new set of orthogonal basis vectors while removing correlation and redundancy information by rotating the basis vectors of the original logging data. The main function of PCA is providing the optimal representation of the original data set by reducing the data dimension. PCA has been widely used in geostatistics to reduce redundancy and dimensionality in the log data. PCA reorients this information into linearly independent principal components along the directions of maximum variance. Suppose the dataset $\left(y_{1} ; y_{2} ; \ldots ; y_{b}\right)$ represents a set of $r$-dimensional samples $\left(x_{1} ; x_{2} ; \ldots ; x_{b}\right)$, the transformation vector is denoted by $p$, and $y_{m}=p^{T} x_{m}$. The objective function of PCA is as follows:

$A=\max _{p} \sum_{m-1}^{b}\left(y_{m}-\bar{y}\right)^{2}=\max _{p} p^{T} M_{p}$ And $\bar{y}=\sum_{m-1}^{b} y_{m}$

where $y_{m}$ is a $r$-dimensional vector, $I$ is an $b \times r$ matrix $\left(P=\left(p_{0}, p_{1} ; \ldots ; p_{r-1}\right)\right)$ and $M$ is the data covariance matrix.

Wavelet analysis decomposes a serial of data into a set of functions like the Fourier transform with the following generalized equation, firstly introduced in the field of signal processing.

$\omega[f(u, s)]=\int_{-\infty}^{+\infty} f(t) \psi_{u, s}(t) \mathrm{d} t$

where $u$ and $s$ are translation and scale, respectively, and $\psi_{u, s}(t)$ is a family of basis functions with the following equation:

$\psi_{u, s}(t)=\frac{1}{\sqrt{s}} \psi\left(\frac{t-u}{s}\right)$

Wavelet transformation can be divided into discrete wavelet transform and continuous wavelet transform (Chu et al. 1996). Because the signal using PCA calculation with mud logger dataset is discrete, it is more suitable to implement a discrete wavelet to decompose it into an orthogonal discrete set of wavelets, which then can be reconstructed without the noise information. The main idea of discrete wavelet transformation is filtering the signal by passing it through high-frequency and low-frequency filters to obtain both the detailed and coarse information of the original data. On the contrary, the reconstruction procedure can be computed from a discrete approximation and a detailed signal at a coarser scale. Overall, decomposition of a data sequence into mutually orthonormal subspaces with wavelet transforms and the corresponding reconstruction procedure are illustrated in Fig. 2.
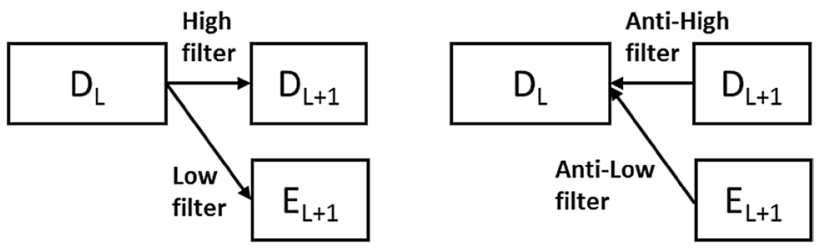

Fig. 2 Wavelet transform of decomposition and reconstruction

Table 1 Component score coefficient matrix

\begin{tabular}{lcc}
\hline & Component 1 & Component 2 \\
\hline ROP & 0.106 & 0.431 \\
WOB & 0.265 & -0.113 \\
RPM & 0.296 & 0.163 \\
SPP & -0.046 & 0.217 \\
TQ & 0.261 & 0.301 \\
Bit time & -0.198 & 0.364 \\
Flow-in & 0.224 & -0.269 \\
\hline
\end{tabular}

\section{Modeling with a sample dataset}

In the case study, the real-time bit dull grade data were not enough to set up the analysis sample; therefore, a linear mode was created to simulate the bit wear in the downhole:

$\Delta \mathrm{BG}=\frac{D_{\text {Current }}-D_{\text {bit_in }}}{D_{\text {bit_out }}-D_{\text {bit_in }}} \times \mathrm{BG}_{\text {bit_out }}$

where $\triangle \mathrm{BG}$ is the IADC bit dull grade in current depth, $D_{\text {Current }}$ is current depth, $D_{\text {bit_in }}$ is running in hole bit depth, $D_{\text {bit_out }}$ is pulling out of hole depth and $\mathrm{BG}_{\text {bit_out }}$ is the IADC bit dull grade after pulling out.

Based on $\triangle B G$ model with the previous data, a study sample was set up and the PCA approach was applied to analyze the mud logger dataset with the following results. Table 1 presents the coefficient matrix for each mud logger dataset, which contains $75 \%$ initiate eigenvalue information of the raw dataset with 55\% eigenvalue in Component 1 and 28\% eigenvalue in Component 2. Figure 3 shows that each member in $\triangle \mathrm{BG}$ is projected into the scatter plot with the two calculated components, with each member not clearly separated as expected. To predict the exact bit wear grade, wavelet analysis is applied into Component 1 with the PCA results.

Based on the analysis experience in the study area, decomposition of the wavelet method obtained valid results at Level 5 as shown in Fig. 4. Figure 4a presents the original signal $s$ with PCA results, low-frequency filter $a_{5}$ at Level 5 decomposition and high-frequency filter $d_{1}, d_{2}, d_{3}, d_{4}, d_{5}$ at all decomposition levels. Figure $4 \mathrm{~b}$, c presents the reconstruction with Level 5 
Fig. 3 The scatter plot with calculated component 1 and component 2

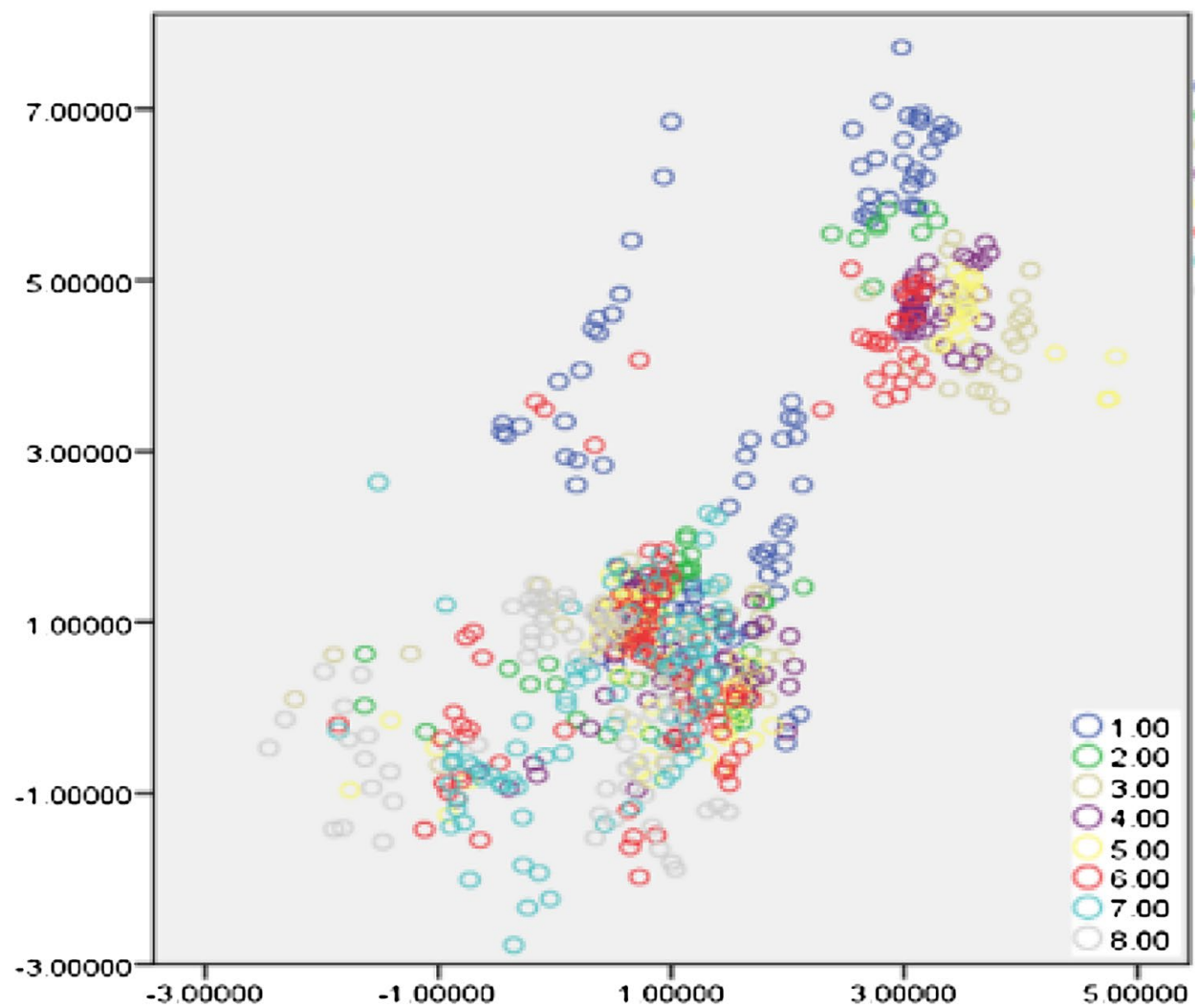

wavelet result, and the basic characteristics of PCA Component 1 of mud logger dataset were obtained.

To obtain the bit wear prediction model, the equation is based on the linear model method with the start point value and the endpoint value as follows:

$\Delta \mathrm{BG}=\frac{\left|S-S_{\text {start }}\right|}{\left|S_{\text {end }}-S_{\text {start }}\right|} \times \mathrm{BG}$

where $s$ is the point required to predict the bit wear dull grade, $S_{\text {start }}$ is the point with wear dull grade value 1 and $S_{\text {end }}$ is the point with wear dull grade value 8 . Based on the study, the value of $S_{\text {start }}$ is set at 75 and the value of $\left|S_{\text {end }}-S_{\text {start }}\right|$ is set at 30.8. The model result with actual bit wear dull grade is discussed in the sample dataset, which shows the accuracy percentage at $42 \%$. The reason for the error rate is primarily the sample dataset obtained with the simple model, which is not the realistic scenario of gradually accelerating the bit wear once bit cutter abrasion starts. Thus, this approach has been applied to each case study to verify the efficiency of this application.

\section{Case study and discussion}

Wavelet analysis method was applied to different drilling section using the Kymera hybrid drill bit in different wells. Figure 5 shows the first case study in Well B where the bit wear prediction on the pulling depth is Grade 5 while the actual dull grade of PDC part is Grade 5-5. As the wavelet analysis result shows, the beginning depth of the predicted bit wear is at Grade 4 and the difference in the predicted and the actual bit wear is a standard deviation due to the controlling weight on bit and other drilling parameters to slow down the wearing rate in the first place.

Wavelet analysis method was applied to predict the low wear section with the Kymera hybrid drill bit to verify the application. Figure 6 shows the case study in Well $\mathrm{C}$ where the bit wear prediction on the pulling depth is Grade 1, while the actual dull grade of PDC part is Grade 1-1. Additionally, GR and RT logging data have been added to the present downhole formation information. For the whole bit life, MSE always presents the low value around baseline, which indicates that the bit stayed in a good condition. The 

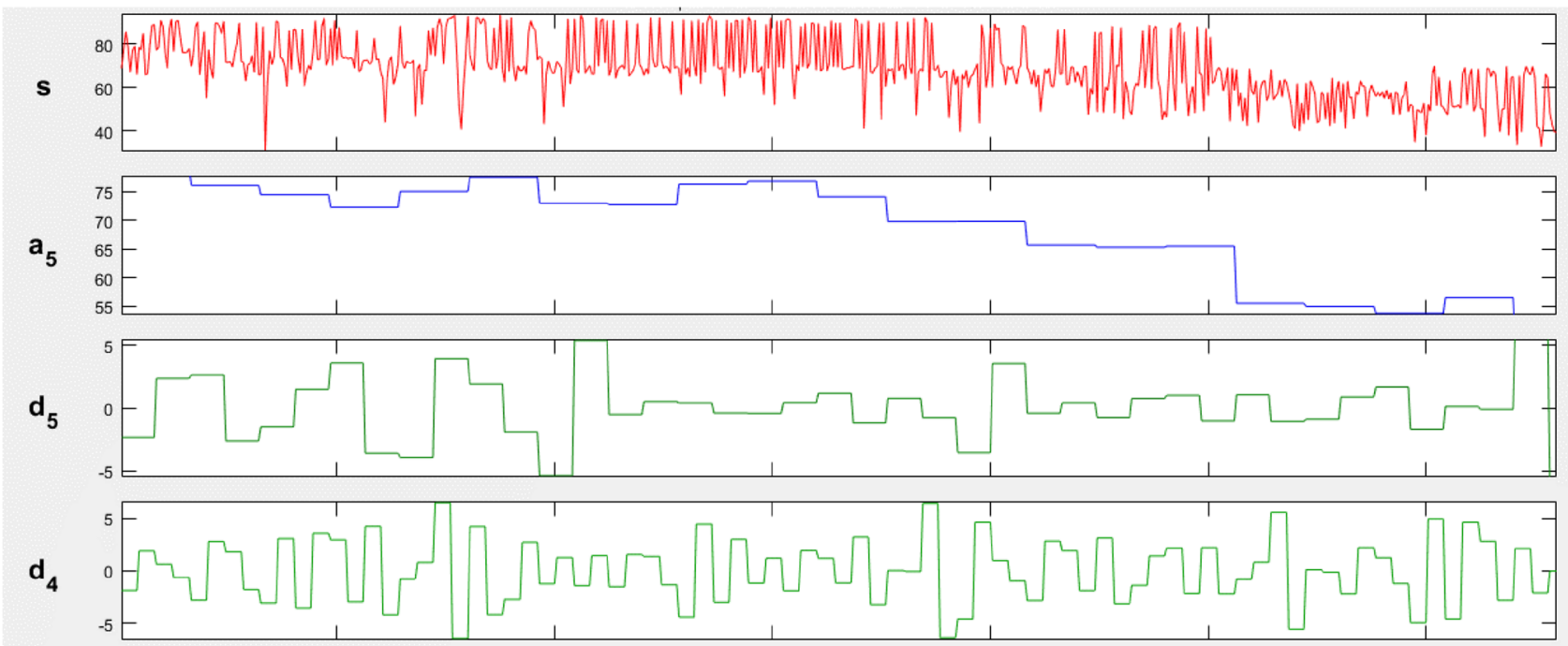

as
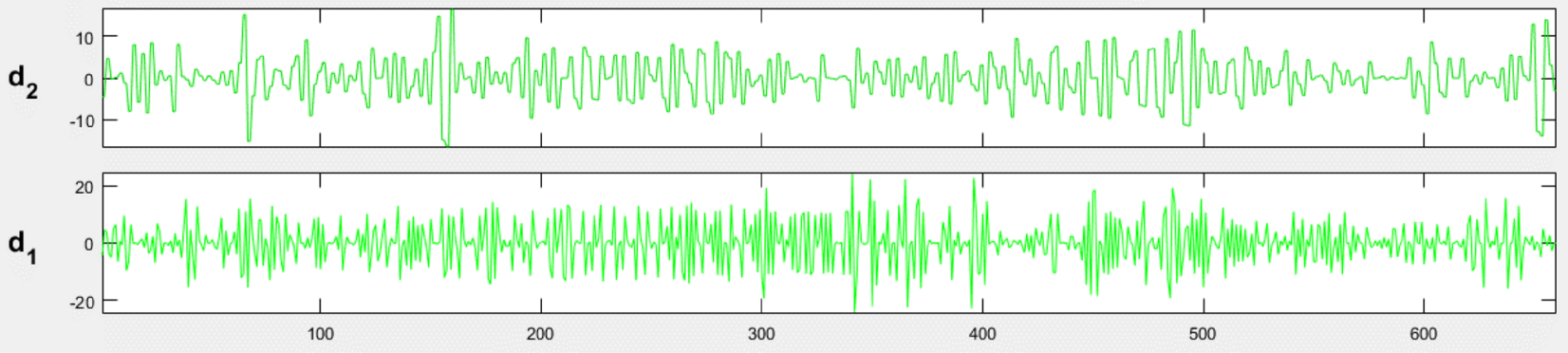

(a) Wavelet decomposition at level 5

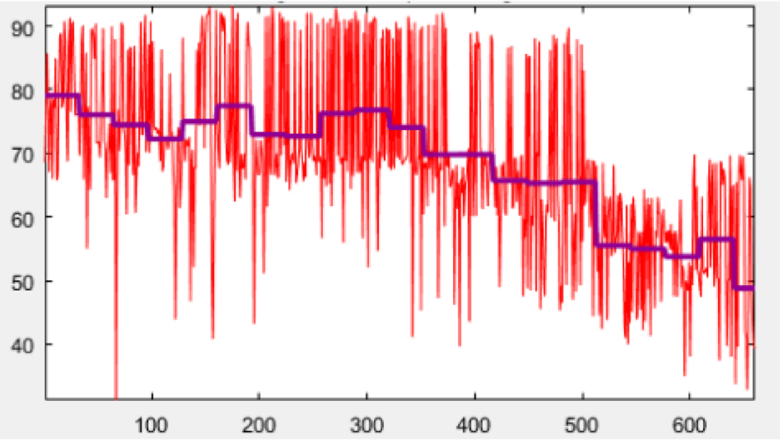

(b) The comparation of original signal and compressed signal

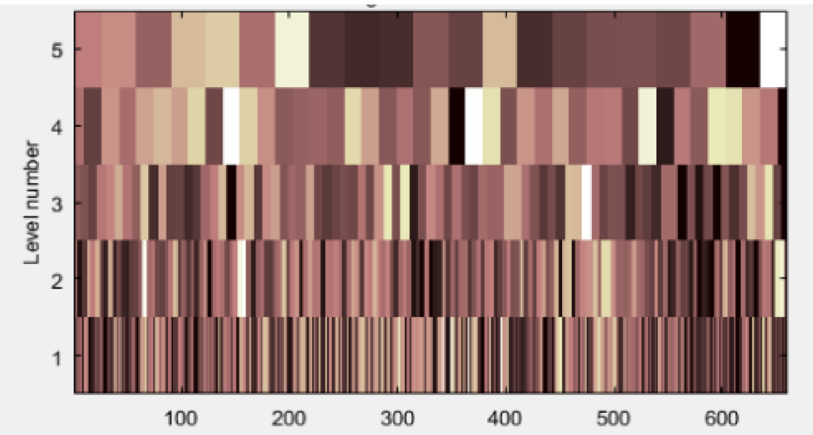

(c) The original coefficients at each level

Fig. 4 Wavelet analysis results with PCA component 1 of mud logger dataset in sample well. a Wavelet decomposition at level 5, b the comparison of original signal and compressed signal, $\mathbf{c}$ the original coefficients at each level

calculation results from 3660 to 3665 m show PDC Grade 2 because a high RPM was applied to the drill. The model considered that it will provide higher friction to the wear bit and it will present back to grade 1 once we slow down RPM. The wavelet analysis, however, precisely predicted the bit dull grade.
This method has been applied in other several wells to testify the accuracy and has received successful prediction rate. The limitation and error analysis show that the sample input dataset is obtained using the linear model instead of the actual bit dull grade due to the limited usage of Kymera hybrid drill bit in the study area and the drill 


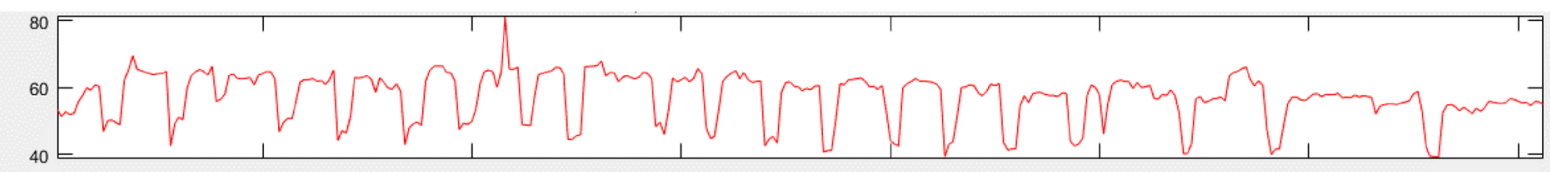

$a_{5}$
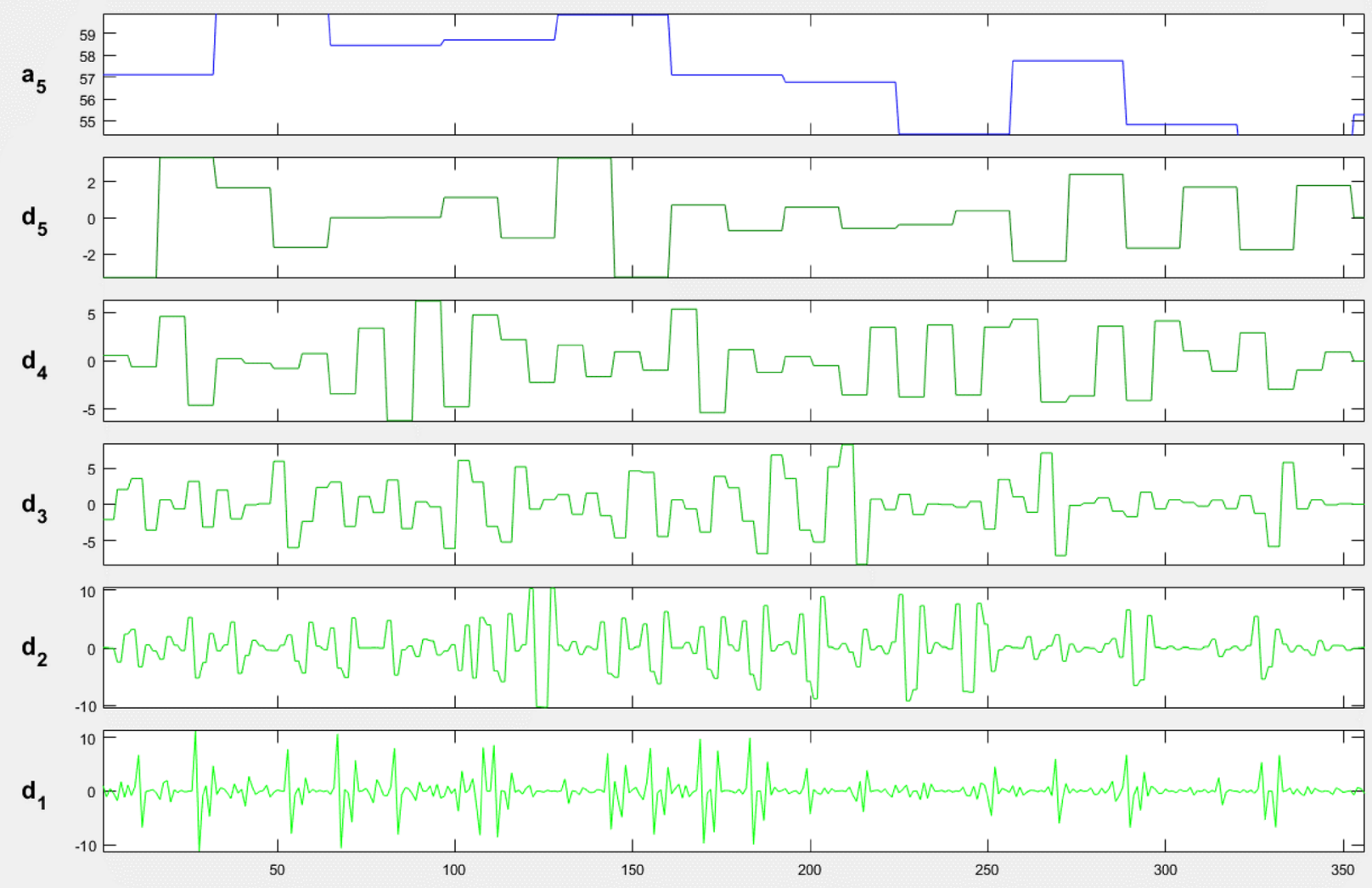

(a) Wavelet decomposition at level 5
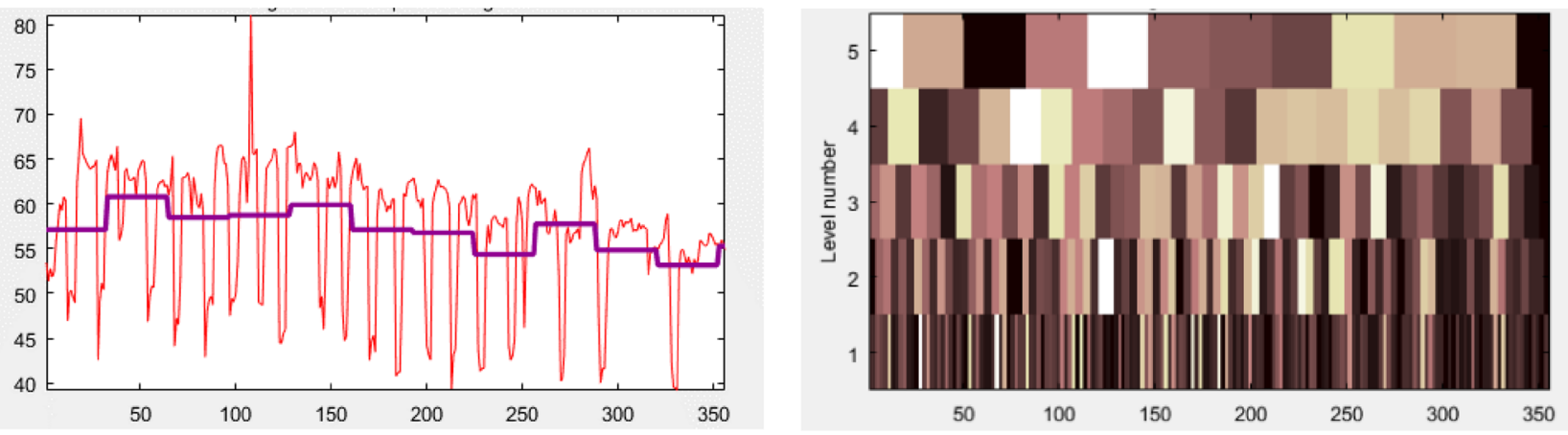

(b) The comparation of original signal and compressed signal

(c) The original coefficients at each level

Fig. 5 Wavelet analysis results with PCA component 1 of mud logger dataset in well B, a wavelet decomposition at level 5, b the comparison of original signal and compressed signal, $\mathbf{c}$ the original coefficients at each level

parameter applied on the new bit always has a large safety margin to reasonably reduce the wearing rate. Thus, the future work is to continue collecting the actual bit dull grade to improve the accuracy of the PCA model and the wavelet method. These methods are not applicable to a new bit drill first section with a large safe margin of limited drilling parameters because the model is mainly built for bit wear prediction with normal drilling parameters. One of the practical applications is to integrate the MSE, PCA and wavelet methods to cross-refer with the driller experience for a more precise, which will obtain the bit wear prediction closed to the reality before being pulled out of the hole. This will ultimately minimize nonproductive time and reduce the number of trips. 


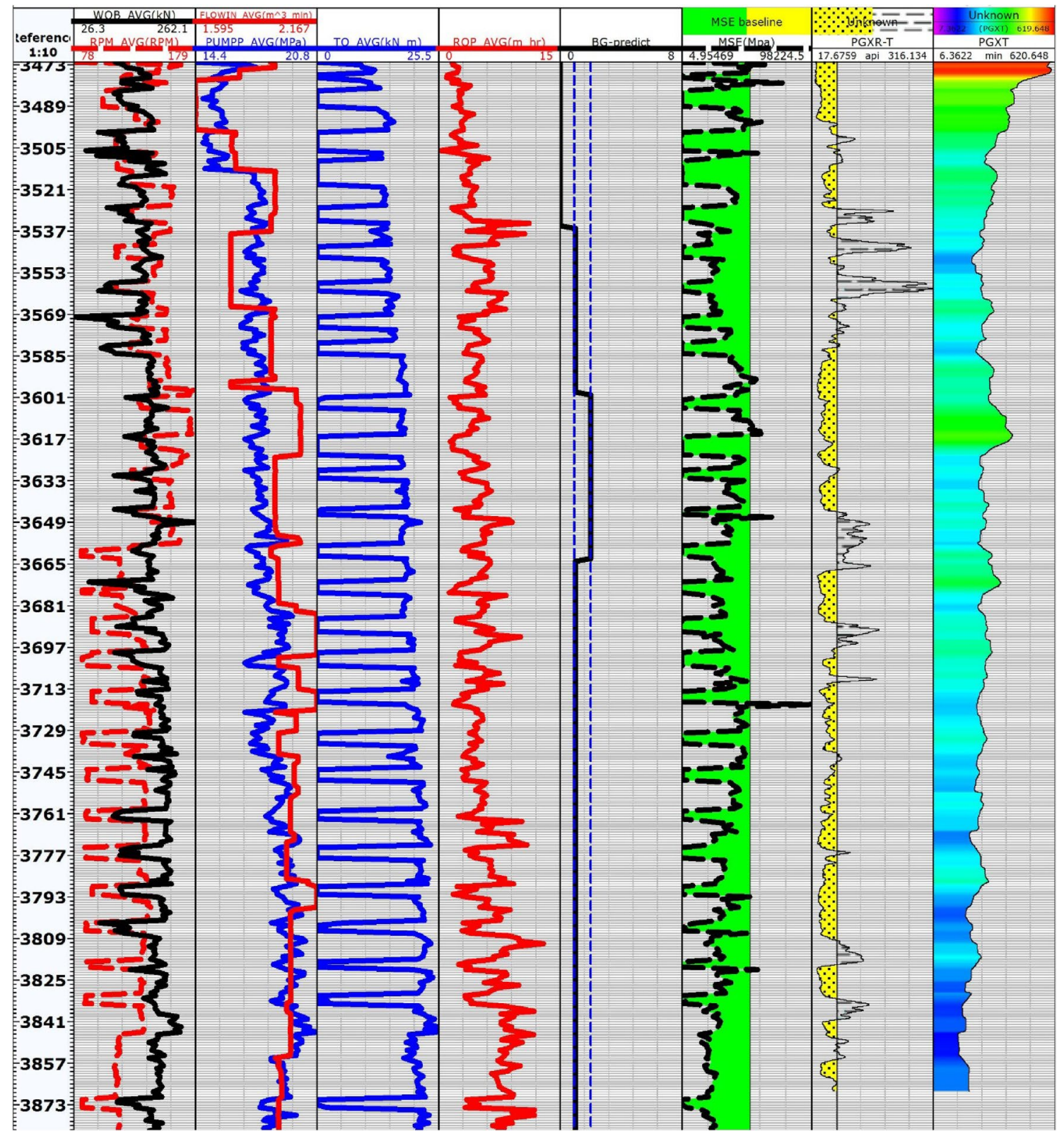

Fig. 6 Wavelet analysis results with PCA component 1 of mud logger dataset in well C

\section{Conclusion}

In this study, traditional methods like MSE and numerical analysis were introduced to real-time bit wear prediction with surface mechanics data. The multivariate data from the mud logger were separately calculated to present the current mechanical energy to monitor downhole drilling efficiency using the MSE method, reduce mud logger data dimension using the PCA method, compress the signal obtained from PCA using wavelet analysis and predict bit wear grade using the model method. Several case studies have been presented in this study to illustrate the advantages of the aforementioned methods and demonstrate the effectiveness of the bit wear prediction. These approaches enable realtime monitoring and bit wear grade prediction for timely and informed decisions while drilling, thereby minimizing nonproductive time by reducing the number of trips.

Open Access This article is distributed under the terms of the Creative Commons Attribution 4.0 International License (http://creativeco mmons.org/licenses/by/4.0/), which permits unrestricted use, distribution, and reproduction in any medium, provided you give appropriate credit to the original author(s) and the source, provide a link to the Creative Commons license, and indicate if changes were made. 


\section{References}

Bourgoyne AT, Young FS (1974) A multiple regression approach to optimal drilling and abnormal pressure detection. J SPE 14(04):371-384

Chu L, Schofzinger RA, Tham MK (1996) Application of wavelet analysis to upscaling of rock properties. In: SPE annual technical conference and exhibition held in Denver, October 1996

Dupriest FE, Koederitz WL (2005) Maximizing drill rates with realtime surveillance of mechanical specific energy. In: Paper SPE/ IADC 92194 presented at the SPE/IADC drilling conference., Amsterdam, The Netherlands, 23-25 February 2005

Motahari HR, Hareland G, James JA, Bartlomowicz M (2008) Improved drilling efficiency technique using integrated PDM and PDC bit parameters. In: SPE gas technology symposium, June 2008, Calgary, AB, Canada

Pessier RC, Hughes Tool Co, Fear MJ, Exploration BP (1992) Quantifying common drilling problems with mechanical specific energy and bit-specific coefficient of sliding friction. In: 67th annual technical conference and exhibition of the society of petroleum engineering, Washington, DC, October 4-7, 1992

Rashidi B, Hareland G et al (2010) Real-time bit wear optimization using the intelligent drilling advisory system. In: Russian oil \& gas technical conference and exhibition held in Moscow, Russia, October 2010

Teale R (1965) The concept of specific energy in rock drilling. Int J Rock Mech Min Sci Geomech Abstr 2(1):57-73

Waughman RJ, Kenner JV, Moore RA (2003) Real-time specific energy monitoring enhances the understanding of when to pull worn PDC bits. SPE Drill Complet 18(1):59-68

Publisher's Note Springer Nature remains neutral with regard to jurisdictional claims in published maps and institutional affiliations. 